\title{
Vehicle Object Retargeting from Dynamic Traffic Videos for Real-Time Visualization
}

\author{
Simon Walton, Kai Berger, David Ebert, Min Chen
}

Citation Information:

S. Walton, K. Berger, D. Ebert and M. Chen, "Vehicle object retargeting from dynamic traffic videos for real-time visualisation,"

The Visual Computer, Springer, 30(5):493-505, 2014.

the date of receipt and acceptance should be inserted later

\begin{abstract}
One form of video visualisation is to transform traffic videos from a street view to an aerial view, which facilitates a summary overview of multiple traffic video streams. This paper presents an efficient and effective solution to mitigate the undesirable distortion of the retargeted vehicle objects in traffic video visualisation. This is achieved by a series of automated algorithmic steps, including vehicle segmentation, vehicle roof detection, and non-uniform image deformation by applying a second homography. This technique has been integrated into a video visualisation system that creates an aerial view of retargeted video streams on top of conventional aerial view. The results have shown that the technique offers the system a significant improvement in visual quality without undermining the requirement for realtime video visualisation.
\end{abstract}

\section{Introduction}

In visual computing, retargeting is a family of techniques for transferring an object or attributes of an object from one scene to another. These techniques support a broad range of image synthesis: for instance, including image and video retargeting for displaying large images on mobile phones (e.g., $[1,2])$, for content adaptation in drama production process [3]; scene relighting (e.g., [4]); motion retargeting and expression retargeting in computer animation $[5,6]$; and design and style retargeting [7,8].

This paper is concerned with the problem of vehicle object retargeting in the context of traffic video visualisation. One particular form of video visualisation requires the synthesis of an aerial view (i.e., helicopter or satellite view) from a camera view [9]. Such a visualization enables users to build up a mental overview of the information captured

Address(es) of author(s) should be given

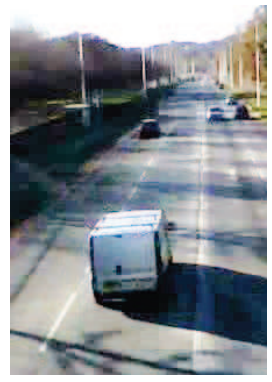

(a) Input

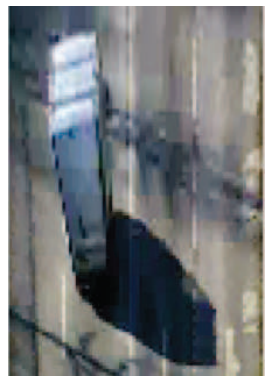

(b) Retarget (naïve)

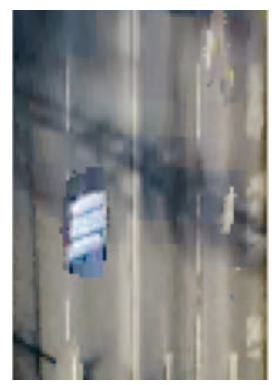

(c) Retarget (Ours)
Fig. 1: Object retargeting in traffic video visualisation scenarios (e.g., from camera view footage to a aerial view of the same scene) with naïve homography-based retargeting leads to severe distortion effects in (b). We make use of optical flow to assist in segmenting the roofs of vehicles and in deriving a second homography to mitigate the distortions in (c).

on multiple traffic video streams in an intuitive manner. One difficulty in creating such visualization is the unknown deformation to be performed on the moving vehicle objects that are being retargeted from the original traffic video to a new visualization. As illustrated in Figure 1, the white van captured on the video appears to be badly distorted in (b) after being retargeted onto the new visualization based on a aerial view. Although users can mentally correct such distortion relatively at ease during visualization, it is still highly desirable to correct such unwanted distortion visually as continuing watching poor quality visualization would burden users with unnecessary cognitive load and possibly stress. One may suggest that such a problem could be solved by re-constructing a $3 \mathrm{D}$ object of the vehicle from multiple camera views, and re-rendering it from the aerial view. However, in most systems of this form of video visualisation, 
users are usually restricted by limited availability of cameras. Hence it is more practical to assume each vehicle may only be captured on a single camera at a time. In addition, it is often necessary to synthesize visualization from dynamic traffic video streams in real time, and hence the computation cost must be kept as low as possible. In this paper, we present a novel technical solution to mitigate this problem in the context of traffic video visualisation. Building on a traffic video visualisation system [9], the solution makes use of a series of automated analytical steps to segment each vehicle from the background, estimate the top plane of a 3D bounding box of the vehicle, and apply a second homography to the vehicle when it is retargeted onto the new visualization.

The results show that not only can this technique mitigate distortions of almost all typical retargeted vehicles in traffic video visualisation, but also can be performed in real time. In the remainder of this paper, we first provide a brief overview of the related works in Section 2, focusing on aspects of video visualisation, retargeting in visual computing and the relevant advances in video processing. In Section 3, we summarize a system that retargets traffic video footages from camera view to a planar visualisation on top of a conventional aerial view such as a satellite image. In Section 5, we describe the effects and causes of distortion in video object retargeting and we detail the technique for distortion mitigation. This is followed by Section 6 where we present and discuss a set of results. We offer our concluding remarks in Section 7.

\section{Related Work}

The transformation of camera view video footage to cartographic aerial view can be classified as an image retargeting operation on the input footage. Image retargeting addresses the task to resize an image to a different size regardless of the resulting aspect ratio and is usually implemented by minimizing a formulated energy functional [10]. In this context, Liu et al. [11] presented a method to intelligently crop video streams for mobile phone systems while allowing the user to interactively pan and resize the crop window. Carroll et al. [12] retarget images in order to change the perspective in the image artistically. Their energy functional is quadratic in the size of the retargeted vertex positions. In another approach, Sacht et al. [13] retarget images from perspective views to cylindrical views. Video visualisation was first proposed by Daniel and Chen [14] as a means for summarization a video segment into a single static image, and was later formalised by Botchen et al. [15]. Chen et al. [16] confirmed that using this kind of summarising, ordinary users can learn to detect and recognise visual signatures of events from video visualisation. Wang et al. [17] proposed to combine videos with a 3D environment model to support situational understanding. This can be considered as a simple form of retargeting of an entire video stream. Remero et al. [18] investigated the visualisation of activities captured by aerial view in natural settings. Legg et al. used homographic projection to reconstruct relatively simple 3D scenes from a single camera view [19] and Parry et al. applied this technique to sports video visualisation [20]. Botchen et al. selected image frames in a video streams and retargeted them into a summary visualization, using VideoPerpetuoGram as the background context [21]. Höferlin made use of VideoPerpetuoGram in sports video visualisation [22]. Borgo et al. provided a comprehensive survey on video-based graphics and video visualisation [23]. There are many types of sensory device for collecting real-time road traffic information. Hoummady provided an excellent set of comments on the shortcomings of each type of sensory device in [24], and also proposed the use of video cameras as data sources for road traffic management. His proposal relies primarily on a computational device that is capable of analysing the scene and recognising 'vehicles, pedestrians, 2-wheel vehicles, etc.' automatically. The proposal does not include any detailed algorithmic solution to the traffic video footage analysis, which remains to be a very challenging technical problem in computer vision; nor does it involve the use of any visualisation techniques. There has been a huge amount of effort in developing techniques for scene analysis and object recognition in the context of traffic video footage processing. Zhu and Li [25] used a simple threshold-based background model for tracking moving vehicles, combined with smoothing filters. Vibha et al. [26] gave a vehicle-counting system, based on counting connected components of the background mask (also using a threshold-based background model). Using background models such as Gaussian Mixture Models to survey moving objects presents issues with the learning rate adopted that governs how quickly the model reacts to newlybackground areas of the image. With a slower learning rate, moving objects tend to leave a trail of pixels that are struggling to learn about the background left in the objects' wake. Many specialised algorithms have also been developed for traffic video footage, such as identification of ground-plane homographies [27]. However, the state of the art frameworks rely heavily on 'two types of a priori information: 1) the contextual information of the camera's field of view and 2) sets of predefined behaviour scenarios [28]. As semantic specifications at different cameras vary significantly, it is difficult to port an automatic solution from a laboratory camera to a large number of real world cameras. Because it is problematic (or at least time-consuming) to specify such semantic information for each individual camera, using automatic computer vision to gather information from videos is yet to become a practical solution. This work focuses on the 


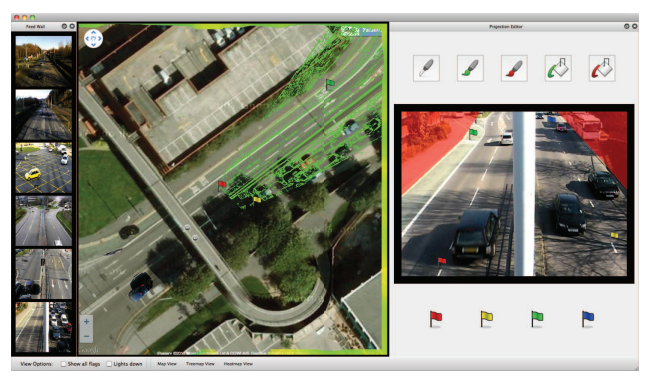

(a)

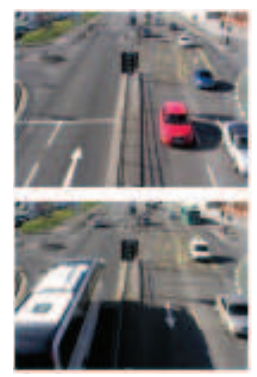

(b)

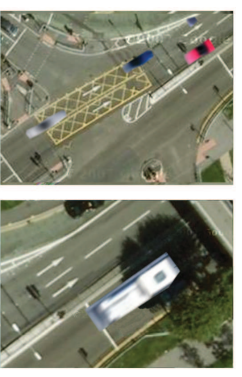

(c)

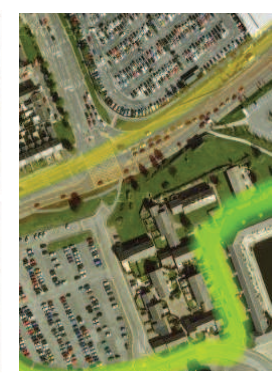

(d)

Fig. 2: The graphical user interface [9], showing the user creating a homography using 'Flag \& Cut' (a). Some traffic video footage (b) is retargeted for visualisation in aerial view (c, distortion mitigation applied). Additionally, the current traffic flow (based on pixel occupancy) may be rendered as a heatmap atop the aerial view (d).

problem of mitigation of retargeting errors. It is beyond the scope of this work to address the challenges in computer vision. We thus built on a traffic video visualisation system [9] that takes an approach that does not rely on automatic scene analysis and object recognition, and provides an easy-to-use user interface for entering necessary contextual information to generate dynamic visualisations without involving object and event recognition.

The most commonly-used convention for road traffic video visualisation is to colour-code lines or areas that represent roads on a map (e.g., [29]). Other traditional visual forms, such as time series plots and heatmaps (without using geographical maps) have also been used to visualise traffic information (e.g., [30]). Ang et al. [31] gave a visual analytical approach to traffic surveillance from multiple cameras with feature extraction to estimate vehicle trajectories.

\section{System Overview}

We are building on a traffic video visualisation system [9], shown in Figure 2, that addresses the following technical goals:

1. A video typically captures the perspective projection of a real-world scene, which is referred to as camera view. The system has to retarget the video data onto a single aerial view, i.e. a 2D plane, with imagery information corresponding to the video.

2. The system has to remove the non-traffic (or background) information on the road in the input footage.

3. The object retargeting has to be performed in real-time.

4. The system has to combine multiple traffic feeds from different camera views onto a single aerial view.

State-of-the art sensor based traffic monitoring systems (e.g., fibre-optic, piezo electric and inductive loops, infrared, laser and Bluetooth-based sensors, GPS, mobile phone data, and weight-in-motion) usually provide relatively accurate information about numbers of vehicles, but their deployment is usually restricted by the shortcomings of each type of sensor: for instance, weight-in-motion sensors and loop sensors require intrusive installation on the road, and are difficult to deploy in ungated open space (e.g., some large car parks). Above-ground optical and electromagnetic sensors (excluding videos) have limited signal ranging, are erroneous in measuring complex traffic flows, and confine real-time traffic video visualisation to a limited set of visual designs - typically colour-coded lines or textual annotation on maps. The above-mentioned aspects were implemented in the functional order of a typical visualisation pipeline (Section 4): the first functional module focuses on data filtering and enrichment, transforming the raw pixel data to more meaningful information. The second module handles the necessary geometric transformation, mapping visual information from camera view to $2 \mathrm{D}$ planar space (i.e., pseudo aerial view). The third module renders the visual information from a pseudoaerial view, and performs image-space composition with a new image background of a conventional aerial view. The three functional modules form a pipeline for generating a video-like layer rendering. In addition, the system provides an alternative pipeline for rendering an estimated traffic density information in aerial views, which we are not discussing furthermore.

\section{Video Object Retargeting}

Let $\mathscr{I}=\left\{I_{1}, I_{2}, \ldots, I_{n}\right\}$ an incoming video stream, where $I_{t}$ be an image frame at time $t$. Our goal is to select a subset of pixels, $P_{t} \subset I_{t}$, from each frame, such that $P_{t}$ are relevant to the objects of interest, in our case, the vehicle objects. $P_{t}$, which may contain multiple vehicles, is then retargeted in real-time to be displayed as foreground objects in a dynamic visualization with an aerial-view background map. The layer where the foreground objects are displayed is referred to as a live layer. The visualisation framework for the Live Layer is desribed in further detail in Section 4.1. 
A naive way to retarget $P_{t}$ is to apply a homograhic transformation to each pixel $\mathbf{p} \in P_{t}$, resulting $\mathbf{p} \rightarrow \mathbf{p}^{\prime}$. This process is described in Section 4.2. As this transformation can introduce significant distortion, which is unpleasant in viewing the dynamic visualization. We thus introduces a second transformation $\mathbf{p}^{\prime} \rightarrow \mathbf{p}^{\prime \prime}$ to mitigating such distortion. This will be described in Section 5 .

Collectively, the two transformations retarget $P_{t}$ to $P_{t}^{\prime \prime}$, which is displayed in the live layer. Thus the integration of $P_{1}^{\prime \prime}, P_{2}^{\prime \prime}, \ldots, P_{n}^{\prime \prime}$ and the background map yields a dynamic visualization, with animated vehicles on the roads in an aerialview map.

\subsection{Visualiation Framework}

The employed traffic video visualisation system [9] aims to empower human vision by transforming raw data to more meaningful information without relying on vision-based object detection. Its focus lies on data enrichment with pixelbased analysis, i.e., extracting information from and augmenting pixels without relying on any algorithms for understanding the scene and its objects. This approach brings some advantages: ( $i$ ) the pixel-level errors and uncertainty have less impact upon the overall perception than objectlevel errors and uncertainty; (ii) it does not require costly data collection and annotation for supervised training in machine learning (iii) pixel-based data filtering and enrichment requires less computational resources and is highly parallelisable. The employed system only classifies pixels in the video streams as either foreground or background by employing a background model. Any background model has inherent uncertainty, with the definition of 'certainty' tied to the context of the scene. For urban traffic video footage where traffic frequently comes to a halt (e.g., at a set of traffic lights), it is unacceptable for such traffic to be absorbed into the background. Therefore the system accepts this uncertainty and introduces methods to ensure that temporarilystationary vehicles on the layer rendering projection are still visible. This is implemented by attaching uncertainty data to a standard Gaussian Mixture Model (GMM) background model via histogram information. Using histogram information, the system estimates the predominant hue of the road area beneath a camera's projection $\operatorname{road}_{r g b}$ and defines a heuristic that models the certainty of a pixel $\mathbf{p}$ being a foreground pixel:

$\operatorname{certainty}(\mathbf{p})=1-\min \left(\left\|\mathbf{p}_{r g b}-\operatorname{road}_{r g b}\right\|^{2}, 1\right) * t c(\mathbf{p})_{\mu}$

where $t c(\mathbf{p})$ gives the topmost GMM cluster at $\mathbf{p}$, and its $\mu$ property denotes the amount of time that cluster has spent in its current sorted position. $\| \mathbf{p}_{r g b}-$ road $_{r g b} \|$ gives the RGB colour space distance between the video colour at $\mathbf{p}$ and road $_{r g b}$. If a vehicle moves into $\mathbf{p}$ and stops, $\mathbf{p}$ will

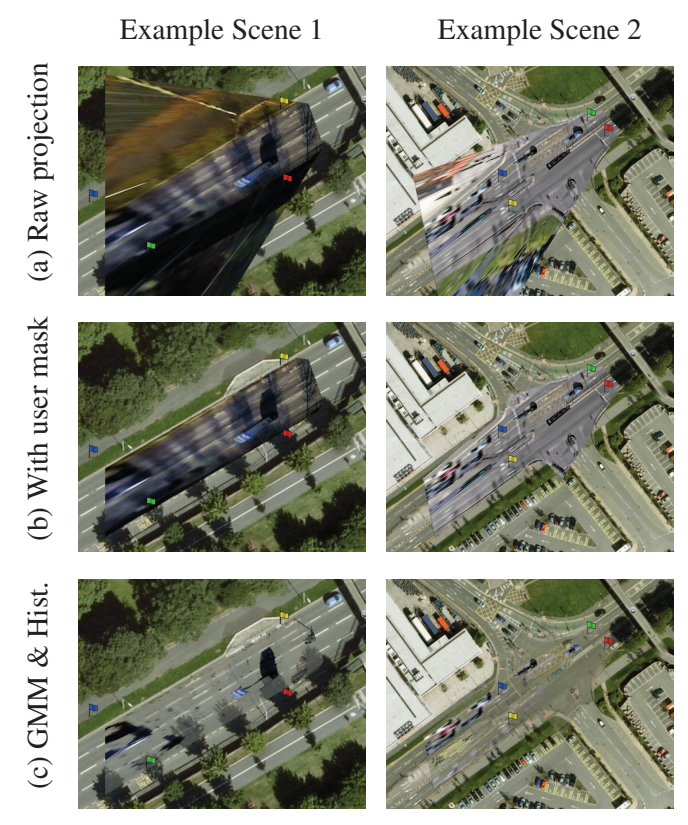

Fig. 3: A comparison of different rendering modes: (a) basic projection of the video onto the map; $(b)$ the projection with the user mask enabled; (c) Gaussian Mixture Model \& histogram matching method, distortions mitigated.

eventually become part of the background. Setting a smaller learning rate can alleviate this somewhat, but at the expense of slower convergence elsewhere. If p's topmost GMM cluster changes frequently, then this probably indicates movement in the area. When a cluster's $\mu$ increases, it becomes less certain as time goes on whether the cluster's pixel represents the road or whether it represents a stationary vehiclethus, the uncertainty can be judged from $\mu$.

\subsection{Homography Definition}

Object retargeting in video visualisation is different from that in most other visualization techniques. Video data contains camera view spatial information; that is, the 3D real real world geometry is represented as projected 2D geometry. Unlike many spatial data types in scientific visualisation (e.g., computed tomography data), the camera view representation in the camera view cannot be directly used by a renderer unless the rendering viewpoint coincides with that of the camera. In the employed visualization system, the basic form of geometry in the final visualisation is predetermined by the requirement for a top-down view emulating a satellite viewpoint. While algorithms exist to automatically extract road features [32], e.g., for providing point correspondences for a homography estimation, the employed visualization system utilises human intelligence instead to define how the video from each camera corresponds to its as- 


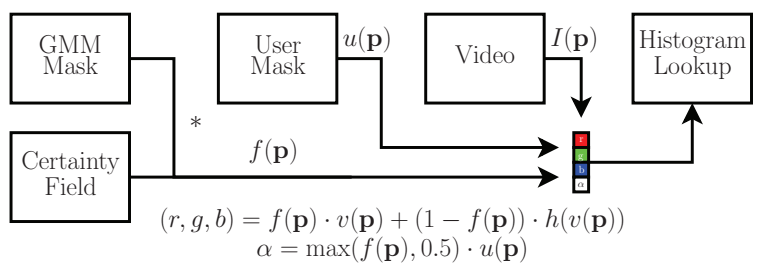

Fig. 4: A pixel p's final fragment colour is decided upon based on the binary user mask $u(\mathbf{p})$, the Gaussian Mixture Model binary mask multiplied by the $[0,1]$ foreground mask $f(\mathbf{p})$, the video frame $I$, and the histogram lookup table $h(\cdot)$.

sociated projection on the map. The three aspects of this definition are:camera view, aerial view, and vehicle.

The user first initiates the definition of a new camera to the system by locating an available camera feed on the feed wall and dragging it into the main aerial view, where the system places the camera on the map (represented by a small camera icon). Once a camera is dropped, the projection editor opens for the user to begin the process of defining four point correspondences of the homography $H_{\text {planar }}$ for the camera, e.g., distinctive lane marks or signposts.

We use a point correspondence system to compute the homography. The corner points for the defining polygon are shown to the user as small flags of colours red, yellow, green, and blue. It is relatively trivial for the user to learn to find places on the video image that correspond with places on the satellite image given in the main map view we have found that cues such as lane arrows, box junction hatches, signposts, lampposts and other road markings provide useful flag positions. There exists the possibility of discrepancies in the video image obtained from the camera and the satellite imagery provided on the map due to out-of-date satellite imagery or obstructions. In such cases, the user relies more on visual feedback to converge to a suitable result.

Inaccuracies in the homography estimation be visible in the visualization phase afterwards, but the relative point locations remain sufficiently accurate after retargeting for traffic monitoring by a human user. The point correspondences, however, may be replaced at any time (see Figure 2).With the homography computed, the user can begin to define which portions of the video are active, and which are inactive. By inactive, we imply regions of the input video that provide no benefit when projected onto the map (pavements, sky, etc). We provide three tools for this: (i) the scalpel for cutting polygonal regions away; (ii) the brush for brushing regions; and (iii) the bucket for the contiguous flood-fill of regions. All tools can function in both video-space and mapspace.

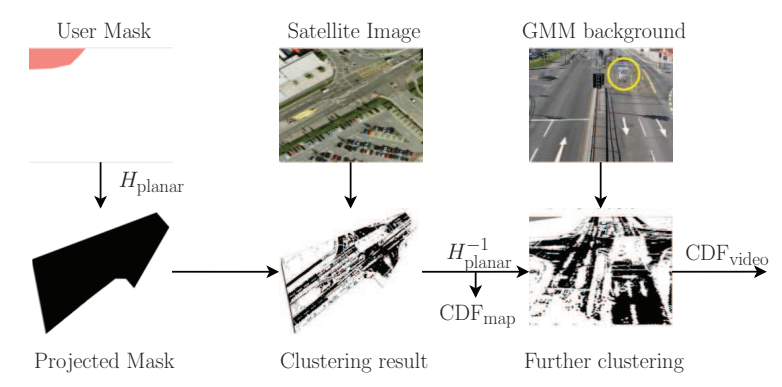

Fig. 5: Computing the histograms to be used for histogram matching. Black pixels represent the active area and white pixels represent the inactive area.

\subsection{Visual Mapping Strategy}

The employed traffic video visualisation system adapts a histogram matching method to blend the projections of the objects onto an existing satellite map. The source histogram is the histogram of the road of the video, and the target is the histogram of the road of the aerial view. It determines automatically which pixels of the video and which pixels of the aerial view are likely those of the road surface (obstructed by vehicles occasionally), see Figure 3 .

The rendering strategy utilises the information on background certainty by favoring pixels that have a high certainty of being foreground over pixels that have a lower certainty of being foreground: Given a pixel $\mathbf{p}$ in camera view related to some point in aerial view, the system associates three attributes:

- A user mask $u(\mathbf{p})$ with binary value $\{0,1\}$ where 0 corresponds to 'inactive' and 1 corresponds to 'active';

- A certainty field $c(\mathbf{p})$ with values $[0,1] \in \mathbb{R}$ ranging from 0: 'uncertain' to 1: 'certain';

- A foreground mask $f(\mathbf{p})$ (obtained from the Gaussian Mixture Model) with binary value $\{0,1\}$ where 0 : 'background' and 1: 'foreground'.

The system then computes the final fragment colour and alpha values as in Figure 4. The histogram function $h(\cdot)$ matches only a limited colour range to blend the video road colour into the map road colour. Note that the colour distance of the input colour to the predominant road colour is already taken into account in the certainty field. Occasionally we find that this strategy can partially fail where a car of similar colour to the road absorbs into the background, but we accept this uncertainty given the relatively low level of incidence.

Figure 5 gives an overview of the histogram matching computation. First, the mask is projected onto the map using homography $H_{\text {planar }}$ to remove unneeded scene regions. Next, a clustering algorithm is applied to the pixel colour values of the underlying road surface in aerial view to ascertain the most frequent colour cluster that is found on the 
scene. A cluster's weight is biased according to the ratio of its RGB values:

$$
\left(1-\left(\left|I(\mathbf{p})_{r}-I(\mathbf{p})_{g}\right|\right)\right) \cdot\left(1-\left(\left|I(\mathbf{p})_{b}-k \cdot I(\mathbf{p})_{b}\right|\right)\right)
$$

where $k=0.94$. The above equation gives a higher weight to pixels that have similar ratios of red to green with a relative $\approx 6 \%$ drop in the blue component. $k$ s 0.94 value has been found empirically by measuring the colour distributions of roads from the satellite imagery: the roads are generally grey but with a drop in blue due to the wavelength of the Suns light.

Once the clustering algorithm completes, a binary mask is created of those pixels on the map belonging to the winning cluster. Once this stage is completed, this mask is projected back onto the video using matrix $H_{\text {planar }}^{-1}$. From the GMM, a 'background' image is obtained by taking the current mean color values of the top distribution for each pixel. Using this background image, a final clustering operation is performed on the video cluster mask using colour information from the GMM background. This final clustering step removes any erroneous information such as shadows being included in the CDF computation. Finally, a RGB histogram
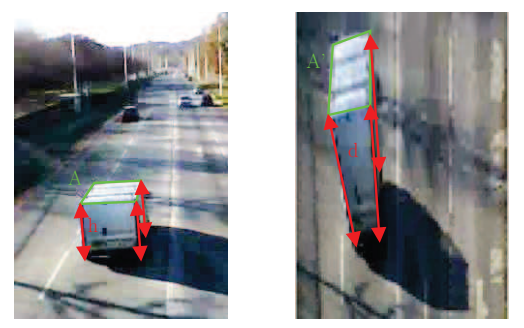

Fig. 6: The distortions $d$ in the aerial view are related to the vehicles' heights $h$. The higher a vehicle the more it gets stretched after retargeting. The area $A$ of a vehicle's roof stays approximately constant $\left(A^{\prime}\right)$.
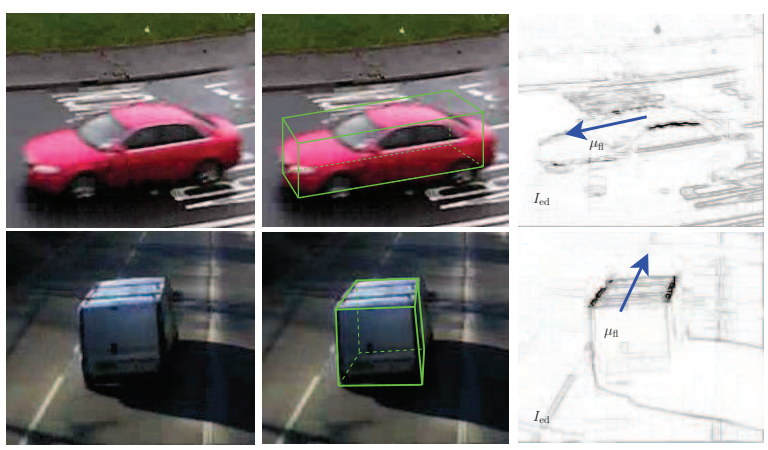

Fig. 7: A vehicle (left column) can be approximated geometrically as a cuboid (middle column) whose top plane correlates to the comprised area in a aerial view. Its side planes usually contribute to distortions. We detect the roof by angular thresholding in $I_{\mathrm{ed}}$ against the mean optical flow $\mu_{\mathrm{fl}}$ (right column). lookup table is computed using the source and target CDFs. In Figure 5 the limitations of tha algorithm are shown, where a blue car present at the beginning of the sequence (circled) has partially absorbed partially absorbed into the background.

To render each camera's projection, the system provides the histogram matching process with the map imagery beneath the projection. The camera's foreground mask $f(\mathbf{p})$, certainty field $c(\mathbf{p})$, current camera frame, and histogram LUT are uploaded to the GPU as textures. The map's projected boundaries are rendered to a bound framebuffer object, and the inverse homography $H_{\text {planar }}^{-1}$ is used in a fragment shader to obtain the final video texture coordinate, discarding the fragment if this coordinate falls outside the $[0,1]$ boundary or if the user mask indicates that the sampled area is inactive. The blending operation shown in Figure 4 is then performed obtain to obtain the final fragment colour. Once all camera views have been rendered to the framebuffer object, the aerial view texture is rendered to screen, and a morphological closure is performed on the final rendering (to reduce 'speckles' in the background model).

We have developed a visual mapping system that implements a conceptually straightforward pixel-based algorithm, mapping directly to an intuitive visualisation that shows the user a real-time estimate of the traffics speed (the amount of foreground change per frame), density, and certainty level. Our algorithm can be viewed as a hybrid of a sensor-based and a video-based input: we treat each projected pixel in the scene as a switch, triggering whenever a car moves over it: Once the density, change, and certainty values have been accumulated, they are normalised by dividing by the total. For a given time window, a rolling mean is calculated for a collection of normalised values of the same type (e.g., density) obtained from the series of consecutive frames in the window. The rolling means for density, change, and certainty are then passed onto the timeline and heatmap visualisation as detailed below. The uncertainty in this system can be classified as validity uncertainty that is, the uncertainty is based on deductive inferences rather than, for example, the quality of the data.

\section{Mitigating Distortions of Retargeted Objects}

Height-related retargeting distortions in the aerial view show severely for objects that move towards the horizon or that move perpendicular to the viewing axis of the camera, Figure 6. The vehicle is usually imaged with its roof and two sides, e.g., back and right side. While the imaged area $A$ of the roof directly relates to the comprised area $A^{\prime}$ of the vehicle on street level, the sides only give sensible information about its height $h$. After applying the homography $H_{\text {planar }}$ the imaged areas of the side mainly contribute of to the perceived distortion $d$ while the roof appears to be displaced. 


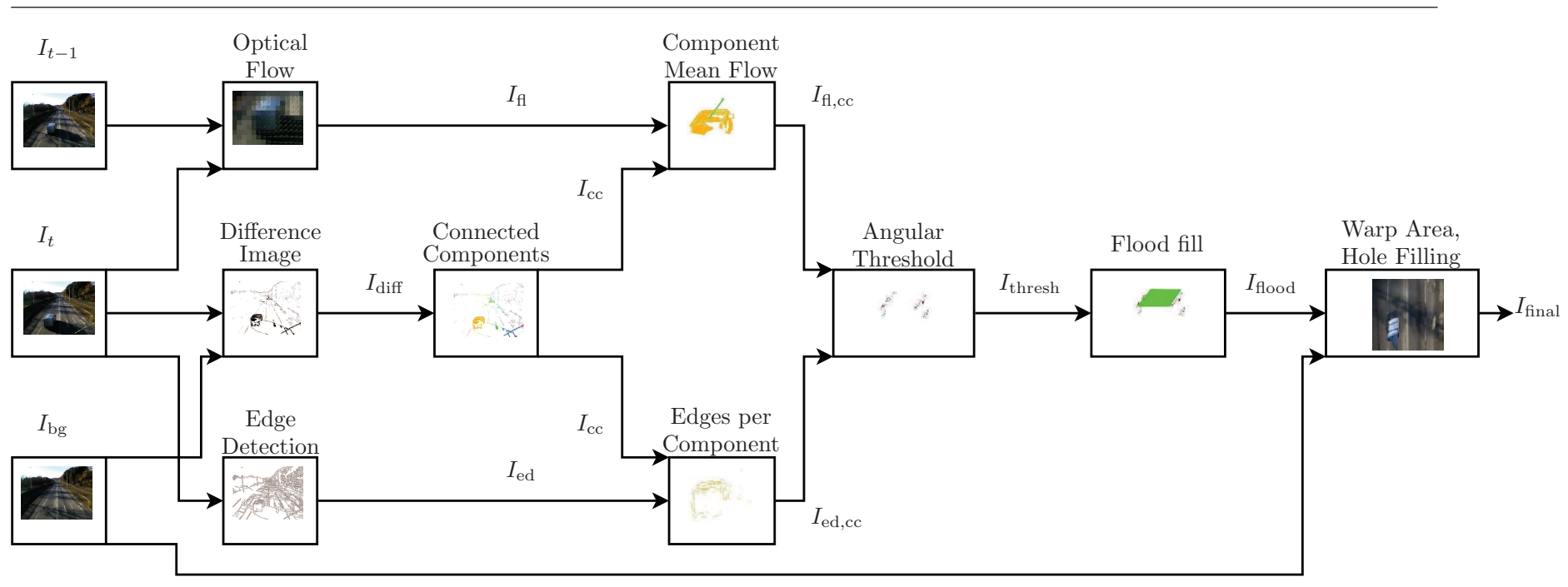

Fig. 8: The flow chart of our retageting algorithm as described in Sect. 5. It takes an average background image $I_{\mathrm{bg}}$ and two consecutive frames $I_{t}$ and $I_{t-1}$ (first column). Then, the algorithm computes the flow field $I_{\mathrm{fl}}$ [33] between the frames (second column, top row) and the connected components $I_{\mathrm{cc}}$ in the current frame $I_{t}$ (third column). As the mean flow vector of the vehicle in $I_{\mathrm{fl}, \mathrm{cc}}$ (fourth column, top row) should roughly align to the edges of a vehicle's roof in $I_{\text {ed,cc }}$ (fourth column, bottom row), an angular thresholding is performed (fifth column). The comprised area of each vehicle's roof is found by a floodfill in $I_{\text {thresh }}$ (sixth column) and then retargeted into the aerial view $H_{\text {planar, height }} \star I_{\text {flood }}$ (seventh column).

Ideally, only the roof, i.e. the top side of an approximated cuboid, should be retargeted into the aerial view. It has to be noted that due to the fact that the roof is located at a height different than the ground plane, the roof has to be retargeted using a different homography $H_{\text {planar, height }} \neq H_{\text {planar }}$. This implicates that the user has to define two different homographies, i.e. 8 point correspondences instead of 4 , to the system, Section 4, for each camera view footage under examination, Figure 9. Note, that a second homography is necessary to avoid large misplacements of the roof plane in the aerial view. In the following we extract the vehicle roof in the input images by assuming:

1. Vehicles can be approximated by cuboids

2. A vehicle roof is planar and rectangular

3. The main axis of its roof aligns to its velocity vector

Assume, that a point $p$ on the edge of the roof of a vehicle object $V$ has a position in world space at times $t$ and $t+1$ defined by $W_{t}(p)$ and $W_{t+1}(p)$, with $W_{*}$ the rigid transformation of the vehicle object to the world space. Let now a camera projection matrix $P$ for a static camera project the point $p$ on the vehicle object $V$ to pixel positions $\mathbf{i}_{t}$ and $\mathbf{i}_{t+1}$ in the camera image with $P\left(W_{t}(p)\right)$ and $P\left(W_{t+1}(p)\right)$. Assume further an adjacent, but visually distinguishable, point $p^{\prime}$ on the edge of the roof of $V$, which is projected to $\mathbf{i}_{t}^{\prime}$ and $\mathbf{i}_{t+1}^{\prime}$ with $P\left(W_{t}\left(p^{\prime}\right)\right)$ and $P\left(W_{t+1}\left(p^{\prime}\right)\right)$. The difference vector in the camera image between $\mathbf{i}_{t}$ and $\mathbf{i}_{t+1}$ can be described by

$\mathbf{d}=\mathbf{i}_{t+1}-\mathbf{i}_{t}$
Further, the projected edge between the adjacent points $p, p^{\prime}$ in the camera image can be described by

$\mathbf{e}_{t}=\mathbf{i}_{t}-\mathbf{i}_{t}^{\prime}$

and

$\mathbf{e}_{t+1}=\mathbf{i}_{t+1}-\mathbf{i}_{t+1}^{\prime}$

for both times $t$ and $t+1$ respectively. There exists a reasonable velocity with which the vehicle moves, so that the point $p^{\prime}$ will be projected at $t+1$ close to the projected position of its adjacent point $p$ at time $t$, thus $\mathbf{i}_{t+1}^{\prime} \approx \mathbf{i}_{t}$ (This holds for the case when $p^{\prime}$ is located further to the rear of the vehicle than $p$ ). Inserting the inequality to Eqn. 1 and Eqn. 3 we get

$\mathbf{d} \approx \mathbf{e}_{t+1}$

We define the residual error of the approximation by the angular difference atan $2(\|\mathbf{d}-\mathbf{e}\|)$. Following the above reasoning, we calculate an optical flow $\mathbf{d}$ between two image frames at times $t$ and $t+1$ and compare it with the edge image at time $t+1$ by computing the angular difference between the two images. Edge pixels with a small residual error are considered as belonging to the vehicles roof. Note, that by calculating the angular difference only, we account for varation in velocity and by taking the absolute diffence we account for the case that $p^{\prime}$ is not located further to the rear of the vehicle than $p$.

Based on the derived Equations, we define the algorithm of our method, Figure 8 as follows: At first segment the vehicles in $I_{t}$ by background subtraction, similar to Section 4, 
and calculates a dense optical flow $I_{\mathrm{fl}}$ [33] between downscaled versions (factor $\frac{1}{8}$ ) of $I_{t}$ and $I_{t-1}$. We use a downscaled version to average out small texture-based deviations in the optical flow image. For each vehicle, i.e. component in $I_{\mathrm{cc}}$, we calculate its mean flow vector $\mu_{\mathrm{fl}}$ to arrive at a $I_{\mathrm{fl}, \mathrm{cc}}$. Then, the edges in the image are compared to $\mu_{\mathrm{fl}}$ for each component in $I_{\mathrm{cc}}$. Edges, which roughly align to $\mu_{\mathrm{fl}}$, are vehicle's roof or bottom boundary. These edges are filtered by angular thresholding to arrive at $I_{\text {thresh }}$. The final area of the roof is computed by performing a flood fill between the remaining edges for each component in $I_{\mathrm{cc}}$. The area is retargeted in to the aerial view with $H_{\text {planar, height }}$ to account for height differences. The missing image parts in the aerial view, which are generated by leaving out the vehicle's sides, are filled with information from the retargeted background image $H_{\text {planar }} \star I_{\text {bg }}$. The long sides are then compared to the remaining velocity vector.

\section{Results}

We tested the proposed algorithm for reducing height-related retargeting distortions with three different traffic scenarios:

- A motorway scene, with traffic flow mainly aligned to the viewing axis;

- A roundabout scene with traffic flow perpendicular to the viewing axis;

- A city bypass scene recorded from a steep angle.

All traffic video footage was acquired using a set of digital camcorders recording video at $640 \times 480$ resolution, at six to twelve FPS. The average height of the bridges is around $20 \mathrm{ft}$, and thus there was much intra-vehicle occlusion in each scene. It should be noted that this work currently focuses only on non-PTZ cameras.

At first we compared different downsampling factors on the input streams for its effect on the accuracy of the mean flow, Table 1. We computed the Average Angular Error (AAE) in degrees between the computed [33] mean optical flow vector and the vehicle's estimated velocity vector in the input stream. It can be seen that a downsampled image averages out small texture-based pixel deviations and the thus more robustly reveals the optical flow introduced by the vehicle's velocity. Best results could be found for a downsampling factor of $\frac{1}{8}$. At that resolution, the vehicles would only span a small amount of pixels in the image and the direction of their velocity vector would dominate the resulting optical flow. Afterwards we compared the amount of distortion on the input sequences in the aerial view for the naïve homography and the homography of our method, Table 2 . The table lists five frames for each input sequence. The first row for each scene in Table 2 shows the input data before downsampling. Note however that the view is cropped partially to

\begin{tabular}{|r|c|c|c|}
\hline \multicolumn{4}{|c|}{ Average Angular Error $\left(^{\circ}\right)$ vs. scale factor } \\
\hline Scale factor & Motorway & Roundabout & Bypass \\
\hline 1 & $5.227^{\circ}$ & $4.070^{\circ}$ & $5.277^{\circ}$ \\
\hline $1 / 2$ & $3.171^{\circ}$ & $3.984^{\circ}$ & $4.234^{\circ}$ \\
\hline $1 / 4$ & $2.661^{\circ}$ & $3.899^{\circ}$ & $3.936^{\circ}$ \\
\hline $1 / 8$ & $1.407^{\circ}$ & $3.504^{\circ}$ & $2.149^{\circ}$ \\
\hline
\end{tabular}

Table 1: We calculated the average angular error (AAE) of the computed mean optical flow $\mu_{\mathrm{fl}}$ [33] to the estimated velocity vector for downsampled versions of the input sequence with different scaling factors. With decreasing input image size, the computed mean flow better approximates the velocity vector by averaging out. This is reasoned by the fact that a downsampled image averages out texture-based pixel deviations.

focus on the vehicles. In the second row for each scene in Table 2 the results for a naïve homography are shown in aerial view. In the third row for each scene in Table 2 the results of our method are shown in aerial view, i.e. a detected vehicle's roof is retargeted with $H_{\text {planar, height, which has been }}$ defined by the user before, Figure 9 . Missing regions in the resulting aerial view are filled with information from a background image $I_{\text {bg }}$ and retargeted with $H_{\text {planar }}$. The fourth row visualizes the differences in the comprised area of the retargeted vehicle: red for a naïve homography and green for our method. It can be seen that, while with the naïve retargeting the comprised area varies with distance to the position of the camera, the comprised area remains more stable with our method (motorway). Also, the comprised area approximates the actual area of the vehicle imaged from a satellite position more accurately as only its roof, i.e. top plane, is retargeted (bypass). Furthermore, the position of the retargeted object appears to be more accurate than with naïve retargeting (roundabout).

\section{Conclusions and Discussion}

We have presented a technique to mitigate distortions in object retargeting in the context of a camera-based global-view traffic video visualisation system [9]. Such a system has the advantage over conventional monitoring systems in that the deployment is not as restricted by the shortcomings of the sensor. However, while such camera-based global-view traffic video visualisation systems directly offer an image of the global traffic situation to the user, they suffer from the fact that the retargeted shape of the visualized objects, varies with distance to the position of the camera. After an overview over the rendering pipeline of the employed traffic video visualisation system, the distortions were identified and their causes were explained. Those distortions were addressed by applying a second homography to the vehicles' 

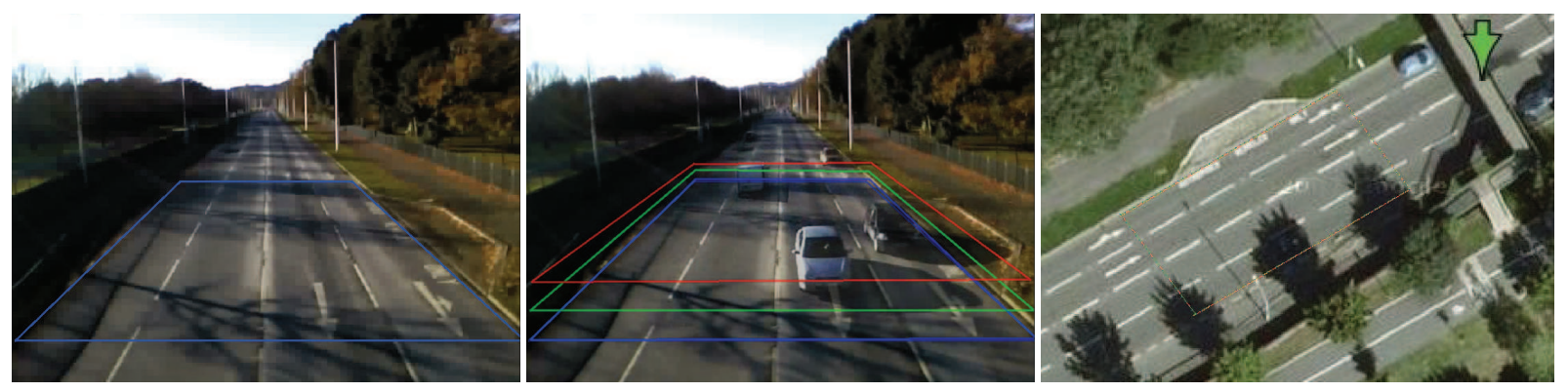

Fig. 9: Homography estimation. Instead of using only one user-defined planar homography $H_{\text {planar }}$ at ground level (left), we propose to use a second user-defined planar homography $H_{\text {planar, height }} \neq H_{\text {planar }}$ (middle) at a height different than the ground level (e.g., car level (green) or lorry level (red)). Note that both homographies, $H_{\text {planar }}$ and $H_{\text {planar, height }}$, map to the same area in the aerial view (right).

roofs which have been detected with a simple real-time capable optical flow-based approach.

We compared our method to the naïve retargeting method in three test scenes. It can be shown that by applying our method the retargeted objects, i.e. the vehicles, were less distorted than with naïve retargeting. There is much scope for future work in this area. The specification of the ground and height homography relies on the video and aerial view providing the same landmarks for registration by the user. In addition, we have concentrated only on aerial views and would like to investigate the interesting challenges that occur when they are replaced with an abstract map. Other challenges include intelligent removing of the existing vehicles on aerial view to arrive at more sophisticated background models.

Also we would like to expand our work from vehicle movements to general object movement scenerios. Such a scenario to test the proposed approach could be intelligent pedestrian surveillance. Ellis and Ferryman for example collected a benchmark database [34] of pedestrian motions on a campus. A combined visualisation of the recorded scene in aerial view, i.e. a rendering of all retargeted camera images promises a good overview and facilitates tracking of single persons. However, as the subjects' heights are usually greater than their comprised area on the ground, the retargeted images are likely to show severe distortions. We think that our method can sufficiently help in mitigating such distortions.

\section{References}

1. Setlur, V., Lechner, T., Nienhaus M. Gooch, B.: Retargeting images and video for preserving information saliency. IEEE Computer Graphics and Applications pp. 80-88 (2007)

2. Michael Rubinstein Ariel Shamir, S.A.: Improved seam carving for video retargeting. ACM Transactions on Graphics (TOG) (2008)
3. Rijsselbergen, D., Poppe, C., Verwaest, M., Mannens, E., Walle, R.: Semantic mastering: content adaptation in the creative drama production workflow. Multimedia Tools and Applications 59(1), 307-340 (2012)

4. Ng, R., Ramamoorthi, R., Hanrahan, P.: Triple product wavelet integrals for all-frequency relighting. ACM Transactions on Graphics (TOG) pp. 477-487 (2004)

5. Gleicher, M.: Retargetting motion to new characters. In: Proc. ACM SIGGRAPH, pp. 33-42 (1998)

6. Hecker, C., Raabe, B., Enslow, R.W., DeWeese, J., Maynard, J., van Prooijen, K.: Real-time motion retargeting to highly varied user-created morphologies. ACM Transactions on Graphics 27(3), 27:1-27:11 (2008)

7. L. Clarke, M.C., Mora, B.: Automatic generation of 3d caricatures based on artistic deformation styles. In: IEEE Transactions on Visualization and Computer Graphics, pp. 808-821 (2011)

8. Lin, J., Cohen-Or, D., Zhang, H., Liang, C., Sharf, A., Deussen, O., Chen, B.: Structure-preserving retargeting of irregular $3 \mathrm{~d}$ architecture. ACM Transactions on Graphics 30(6), 183:1-183:10 (2011)

9. Walton, S., Chen, M., Ebert, D.: Livelayer: Real-time traffic video visualisation on geographical maps

10. Setlur, V., Takagi, S., Raskar, R., Gleicher, M., Gooch, B.: Automatic image retargeting. In: Proceedings of the 4th international conference on Mobile and ubiquitous multimedia, pp. 59-68. ACM (2005)

11. Liu, F., Gleicher, M.: Video retargeting: automating pan and scan. In: ACM Multimedia, pp. 241-250. ACM, New York, NY, USA (2006). DOI $10.1145 / 1180639.1180702$. URL http://doi.acm.org/10.1145/1180639.1180702

12. Carroll, R., Agarwala, A., Agrawala, M.: Image warps for artistic perspective manipulation. ACM Trans. Graph. 29(4), 127:1-127:9 (2010). DOI $10.1145 / 1778765.1778864$. URL http://doi.acm.org/10.1145/1778765.1778864 
13. Sacht, L., Velho, L., Nehab, D., Cicconet, M.: Scalable motion-aware panoramic videos. In: SIGGRAPH Asia 2011 Sketches, p. 37. ACM (2011)

14. Daniel, G., Chen, M.: Video visualization. Proc. IEEE Visualization pp. 409-416 (2003)

15. Botchen, R.P., Bachthaler, S., Schick, F., Chen, M., Mori, G., Weiskopf, D., Ertl, T.: Action-based multifield video visualization 14(4), 885-899 (2008)

16. Chen, M., Botchen, R.P., Hashim, R.R., Weiskopf, D., Ertl, T., Thornton, I.M.: Visual Signatures in Video Visualization. IEEE Transactions on Visualization and Computer Graphics 12(5), 1093-1100 (2006)

17. Wang, Y., Krum, D.M., Coelho, E.M., Bowman, D.A.: Contextualized videos: Combining videos with environment models to support situational understanding. IEEE Transactions on Visualization and Computer Graphics 13(6), 1568-1575 (2007). DOI 10.1109/TVCG.2007.70544

18. Remero, M., Summet, J., Stasko, J., Abowd, G.: Viza-vis: Toward visualizing video through computer vision. IEEE Transactions on Visualization and Computer Graphics 14(6), 1261-1268 (2008). DOI 10.1109/TVCG.2008.185

19. Legg, P., Parry, M., Chung, D., Jiang, R., Morris, A., Griffiths, I., Marshall, D., Chen, M.: Intelligent filtering by semantic importance for single-view $3 \mathrm{~d}$ reconstruction from snooker video. In: ICIP, pp. 2433-2436 (2011)

20. Parry, M., Legg, P., Chung, D., Griffiths, I., Chen, M.: Hierarchical event selection for video storyboards with a case study on snooker video visualization. In: IEEE Transactions on Visualization and Computer Graphics, pp. 1747-1756 (2011)

21. Botchen, R., Bachthaler, S., Schick, F., Chen, M., Mori, G., Weiskopf, D., Ertl, T.: Action-based multi-field video visualization. In: TVCG (2008)

22. Hoeferlin, M., Grundy, E., Borgo, R., Weiskopf, D., Chen, M., Griffiths, I., Griffiths, W.: Video visualization for snooker skill training. In: Computer Graphics Forum, pp. 1053-1062 (2010)

23. Borgo., R., Chen, M., Daubney, B., Grundy, E., Jaenicke, H., Heidemann, G., Hoeferlin, B., Hoeferlin, M., Weiskopf, D., Xie, X.: A survey on video-based graphics and video visualization. In: Eurographics 2011 STAR (2011)

24. Hoummady, B.: Method and device for managing road traffic using a video camera as data source. United States Patent No. US 6,366,219 B1 (2002)

25. Zhu, F., Li, L.: An optimized video-based traffic congestion monitoring system. In: Proc. 3rd International Conference on Knowledge Discovery and Data Mining, pp. 150-153 (2010). DOI http://dx.doi.org/10.1109/WKDD.2010.47. URL
http://dx.doi.org/10.1109/WKDD.2010.47

26. Vibha, L., Venkatesha, M., Prasanth, R.G., Suhas, N., Shenoy, P.D., Venugopal, K.R., Patnaik, L.M.: Moving vehicle identification using background registration technique for traffic surveillance. In: Proc. International MultiConference of Engineers and Computer Scientists, vol. I, pp. 19-21 (2008)

27. Arrospide, J., Salgado, L., Nieto, M., Mohedano, R.: Homography-based ground plane detection using a single on-board camera. IET Intelligent Transport Systems 4(2), 149-160 (2010). DOI 10.1049/iet-its.2009.0073

28. Kumar, P., Ranganath, S., Weimin, H., Sengupta, K.: Framework for real-time behavior interpretation from traffic video. IEEE Transactions on Intelligent Transportation Systems 6(1), 43-53 (2005). DOI 10.1109/TITS.2004.838219

29. Shekhar, S., Lu, C.T., Liu, R., Zhou, C.: CubeView: A system for traffic data visualization. In: Proc. IEEE Intelligent Transportation Systems, pp. 674-678 (2002)

30. Lu, C.T., Boedihardjo, A.P., Zheng, J.: AITVS: Advanced interactive traffic visualization system. In: Proc. International Conference on Data Engineering, pp. 167-168 (2006). DOI http://doi.ieeecomputersociety.org/10.1109/ICDE.2006.14

31. Ang, D., Shen, Y., Duraisamy, P.: Video analytics for multi-camera traffic surveillance. In: Proc. 2nd International Workshop on Computational Transportation Science, pp. 25-30. New York, NY, USA (2009). DOI http://doi.acm.org/10.1145/1645373.1645378. URL http://doi.acm.org/10.1145/1645373.1645378

32. He, Y., Wang, H., Zhang, B.: Color-based road detection in urban traffic scenes. Intelligent Transportation Systems, IEEE Transactions on 5(4), 309-318 (2004)

33. Horn, B., Schunck, B.: Determining optical flow. Artificial intelligence 17(1), 185-203 (1981)

34. Ellis, A., Ferryman, J.: Pets 2010 and pets2009 evaluation of results using individual ground truthed single views. In: AVSS 2010, pp. 135-142. IEEE (2010) 


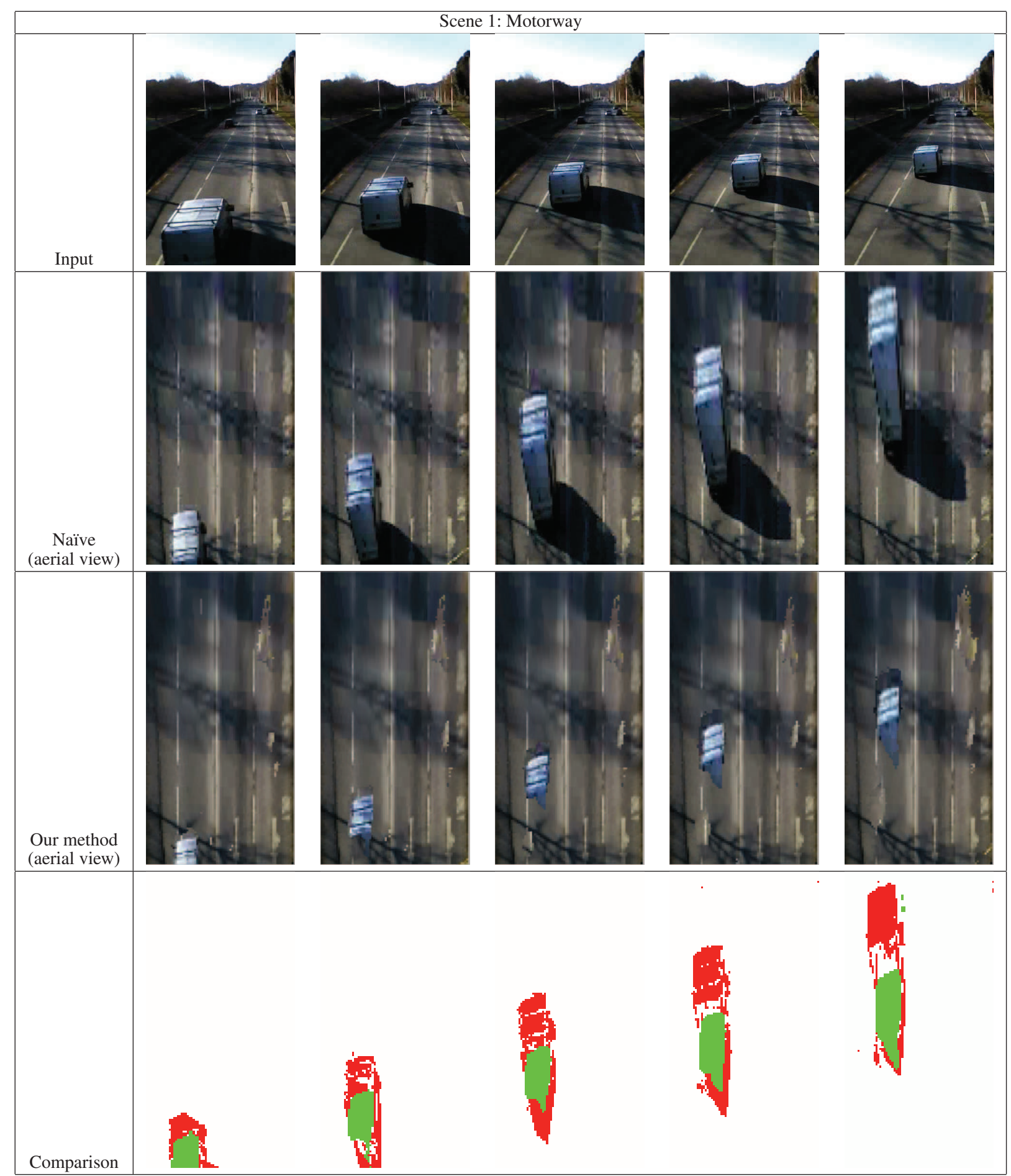

Table 2: Retargeting distortion mitigation applied to three test scenes. The first row for each scene shows the input data before downsampling. The second row for each scene shows the results for a naïve homography in aerial view. The third row for each scene shows the results of our method in aerial view. In the fourth row for each scene the comprised areas are compared for both methods in aerial view (red = naïve, green = our method). It can be seen that the comprised area in aerial view approximates the actual area more accurately with our method. The first scene shows a motorway with traffic flow mainly aligned to the viewing axis. The second scene shows a roundabout with traffic flow perpendicular to the viewing axis. The third scene shows a city bypass with multiple cars recorded from a steep angle. 


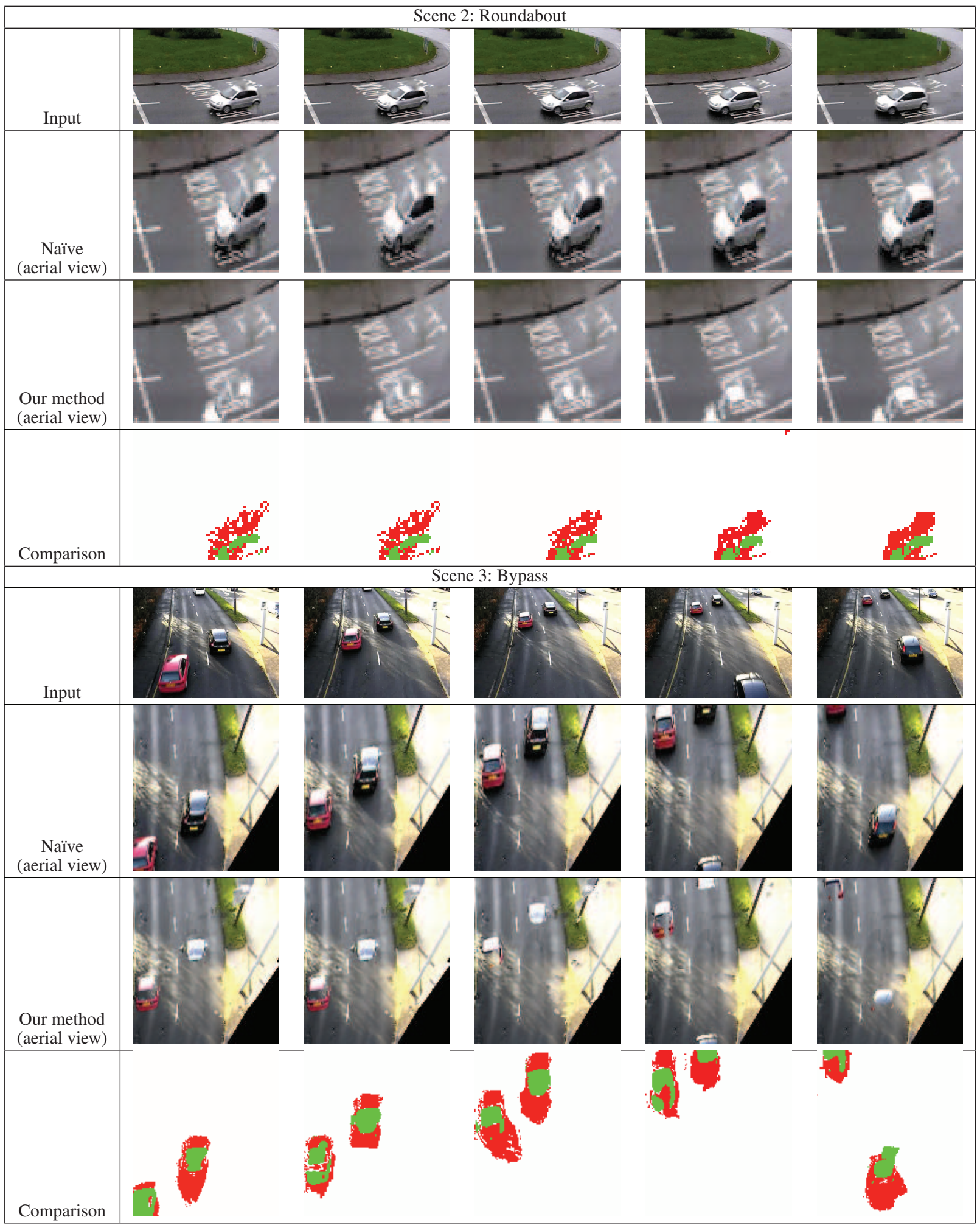

Table 2: (continued) Retargeting distortion mitigation applied to three test scenes (first row each) and comparison (fourth row each, red = naïve, green = our method). It can be seen, that the comprised area approximates the actual area more accurately with our method. The first scene shows a motorway, the second shows a roundabout, and the third shows a city bypass with multiple cars. 